\title{
Powassan Virus: An Emerging Arbovirus of Public Health Concern in North America
}

\author{
Meghan E. Hermance ${ }^{1}$ and Saravanan Thangamani ${ }^{1-3}$
}

\begin{abstract}
Powassan virus (POWV, Flaviviridae) is the only North American member of the tick-borne encephalitis serogroup of flaviviruses. It is transmitted to small- and medium-sized mammals by Ixodes scapularis, Ixodes cookei, and several other Ixodes tick species. Humans become infected with POWV during spillover transmission from the natural transmission cycles. In humans, POWV is the causative agent of a severe neuroinvasive illness with 50\% of survivors displaying long-term neurological sequelae. POWV was recognized as a human pathogen in 1958 when a young boy died of severe encephalitis in Powassan, Ontario, and POWV was isolated from the brain autopsy of this case. Two distinct genetic lineages of POWV are now recognized: POWV (lineage I) and deer tick virus (lineage II). Since the index case in 1958, over 100 human cases of POWV have been reported, with an apparent rise in disease incidence in the past 16 years. This recent increase in cases may represent a true emergence of POWV in regions where the tick vector species are prevalent, or it could represent an increase in POWV surveillance and diagnosis. In the past 5 years, both basic and applied research for POWV disease has intensified, including phylogenetic studies, field surveillance, case studies, and animal model development. This review provides an overview of POWV, including the epidemiology, transmission, clinical disease, and diagnosis of POWV infection. Recent research developments and future priorities with regard to the disease are emphasized.
\end{abstract}

Keywords: deer tick virus, encephalitis, Ixodes scapularis, Powassan virus, tick-borne flavivirus

\section{Introduction}

$\mathbf{P}$ OWASSAN VIRUS (POWV) is a tick-borne flavivirus that can cause a fatal neuroinvasive disease in humans. The virus was first identified as a human pathogen in 1958 when it was isolated from the brain of a young boy who died of encephalitis in the town of Powassan, Ontario (McLean and Donahue 1959). Since then, human cases of POWV have been documented in the United States, Canada, and Russia. Cases in the United States are typically reported in the northeastern and midwestern states. In recent years, the incidence of human infection due to POWV appears to be rising
(Hinten et al. 2008, Ebel et al. 2010, Piantadosi et al. 2016). From 1958 through 1998, only 27 human POWV cases were reported from eastern Canada and the northeastern United States (Gholam et al. 1999); whereas from 2003 to present day, 85 human cases of Powassan encephalitis have been documented in the United States alone (USGS Disease maps: http://diseasemaps.usgs.gov/mapviewer). This recent increase may be due to increased surveillance and testing for arthropodborne viruses, an actual emergence of the disease, or a combination of both factors.

Compared to the other human pathogenic flaviviruses, POWV is not as well studied. Because POWV is classified as

\footnotetext{
${ }^{1}$ Department of Pathology, University of Texas Medical Branch, Galveston, Texas.

${ }^{2}$ Institute for Human Infections and Immunity, University of Texas Medical Branch, Galveston, Texas.

${ }^{3}$ Center for Tropical Diseases, University of Texas Medical Branch, Galveston, Texas.

(c) Meghan E. Hermance and Saravanan Thangamani 2017; Published by Mary Ann Liebert, Inc. This is an Open Access article distributed under the terms of the Creative Commons Attribution License, which permits unrestricted use, distribution, and reproduction in any medium, provided the original work is properly cited.
} 
a member of the tick-borne encephalitis serocomplex of flaviviruses, a large amount of our understanding about POWV replication and its potential to cause disease in humans comes from research conducted with the closely related tick-borne encephalitis virus (TBEV). Each year, TBEV causes several thousand human cases of neuroinvasive illness across Europe and in parts of Asia. With more people traveling to TBEVendemic areas and the increase in disease incidence, TBEV has become an international public health concern (Suss 2011). In 1993 the first complete genomic sequence of POWV was published (Mandl et al. 1993). Similar to the TBEV genome, the POWV genome consists of single-stranded, positive-sense RNA of approximately $11 \mathrm{~kb}$ that encodes a single, long, open reading frame. The $3^{\prime}$ portion of the genome encodes seven nonstructural proteins. Three structural proteins are encoded at the $5^{\prime}$ end of the genome: the capsid (C) protein, the premembrane (prM) protein, and the envelope (E) glycoprotein. Based on antigenic and molecular genetic criteria, POWV is classified as a member of the TBE serocomplex of flaviviruses (Calisher et al. 1989, Zanotto et al. 1996, Kuno et al. 1998).

Since Ebel's comprehensive POWV review published in 2010, additional phylogenetic analyses, serosurveys, case series, animal model studies, and basic science experiments have been conducted for POWV. The present review provides an overview of POWV, emphasizing recent research developments. This review also aims to raise clinician awareness of the disease by providing detailed descriptions of the disease manifestations, pathogenesis, and current diagnostic standards. The final section concludes by discussing future research priorities.

\section{History of Powassan Virus}

\section{Geographic distribution}

After POWV was first recognized as a human pathogen in 1958 , vector and vertebrate surveillance studies were conducted in surrounding regions of Ontario, and it was quickly determined that POWV is a tick-borne pathogen vectored by several Ixodes species ticks (McLean and Larke 1963, McLean et al. 1964, 1967). In North America, the geographic distribution of POWV has been determined by reported human cases and by screening ticks and vertebrate hosts in field studies; however, underdiagnosis of human POWV cases may contribute to our limited knowledge of the actual geographic distribution of POWV (Schotthoefer et al. 2016). On the eastern seaboard of North America, the virus has been detected as far south as Virginia and as far north as Nova Scotia. POWV is also found inland in New York, Pennsylvania, and Quebec (Artsob 1989). Further into the North American interior, POWV has been documented in north-central states such as Michigan, Wisconsin, and Minnesota (Hinten et al. 2008), western states such as Colorado and California (Thomas et al. 1960, Johnson 1987), and broadly throughout the Canadian provinces of Ontario (McLean and Donahue 1959), Alberta (Zarnke and Yuill 1981), British Columbia (McLean et al. 1970), and Quebec (Rossier et al. 1974).

\section{Molecular phylogenetics}

Forty years after the index case of POWV, it became apparent that the population structure of POWV was genetically and ecologically diverse. In 1997, a virus that was similar but distinct from POWV was isolated in New England (Telford et al. 1997). This POWV-like virus was named deer tick virus (DTV) because it was isolated from the Ixodes scapularis tick, otherwise known as the deer tick (Telford et al. 1997). Nucleotide sequencing revealed a high level of genetic similarity between DTV and POWV, with $84 \%$ nucleotide sequence identity and 94\% amino acid identity (Beasley et al. 2001, Kuno et al. 2001). Genomic sequencing demonstrated that POWV consists of two genetic lineages or genotypes: Lineage I, which is the POWV prototype lineage, and Lineage II, which consists of DTVs (Beasley et al. 2001, Ebel et al. 2001, Kuno et al. 2001). Although cross-neutralization studies show that POWV and DTV are serologically indistinguishable, phylogenetic distinctions are evident; thus, POWV and DTV make up two different genotypes of the same virus (Beasley et al. 2001, Kuno et al. 2001).

Molecular epidemiology shows POWV and DTV sequences to be relatively stable over time (Pesko et al. 2010), a finding that supports field evidence of stable, persistent viral transmission in midwestern DTV foci (Ebel et al. 1999, Brackney et al. 2008). In Connecticut, the isolation of two distinct subclades of POWV $40 \mathrm{~km}$ apart suggests that the virus was introduced separately into each environment and is maintained in small, stable foci (Anderson and Armstrong 2012). To determine the mechanisms that led to the divergence of POWV into two phylogenetically distinct lineages, several studies have compared nucleotide sequence data, both within and between lineages (Ebel et al. 2001, Pesko et al. 2010). The McDonald-Kreitman test was used to characterize the divergence between POWV and DTV, providing evidence that the divergence between the two lineages is driven by positive natural selection (Ebel et al. 2001, Pesko et al. 2010). These findings suggest that the natural selectiondriven divergence of the two lineages may be due to the reliance of each lineage on a different tick vector species for natural transmission (Ebel et al. 2001). In nature, POWV (Lineage I) is maintained predominantly by Ixodes cookei ticks, while DTV (Lineage II) is maintained by I. scapularis. To date, POWV phylogenetic studies have been restricted by the limited number of strains available and by analyzing a relatively small portion of the genome.

Recent phylogenetic and biogeographic analyses using fulllength open reading frame (ORF) sequences of tick-borne flaviviruses predicted that the ancestral lineage gave rise to POWV/DTV split from the other tick-borne flaviviruses approximately 12.3 thousand years ago (KYA) (Heinze et al. 2012). These estimates suggest that the ancestral POWV lineages dispersed across the Bering Land Bridge before it was flooded by the Bering Sea approximating 11 KYA. These events would have secluded the ancestral POWV lineages from the Old World tick-borne flaviviruses (Heinze et al. 2012). POWV has also been isolated in the far-eastern Primorsky region of Russia, and it is thought that the presence of POWV in Russia can be explained by a single POWV introduction into Russia over one century ago (Artsob 1989, Leonova et al. 2009, Heinze et al. 2012). The sequence conservation between Russian isolates and the Canadian isolate (LB strain) of POWV is $99.8 \%$, with tight phylogenetic clustering; thus, the limited genetic variability between the Russian and Canadian isolates of POWV resulted in the hypothesis that POWV was introduced into Russia by the import of North American mink during the 1800s (Leonova et al. 2009, Heinze et al. 2012). 


\section{Powassan Virus Transmission}

\section{Vector associations}

POWV is maintained in nature by ixodid ticks and mammalian hosts. Field studies have provided numerous assessments of vector associations for POWV and DTV. In 1952, the first isolate of POWV was made from Dermacentor andersoni ticks collected in Colorado (Thomas et al. 1960). POWV has since been isolated from Ixodes marxi, Ixodes spinipalpus, and Ixodes cookei, while DTV has been isolated from I. scapularis (McLean and Larke 1963, McLean et al. 1964, Telford et al. 1997). A few POWV surveillance studies have incidentally collected tick species such as Dermacentor variabilis and $D$. andersoni, but the enzootic transmission of POWV is yet to be described for any Dermacentor species and it is not known whether Dermacentor is an important vector of POWV (Thomas et al. 1960, Brackney et al. 2008).

\section{Enzootic maintenance}

The earliest studies of enzootic maintenance of POWV were conducted in the vicinity of Powassan, Ontario, where the first human case of POWV was documented. These studies implicated red squirrels (Tamiasciurus hudsonicus) and chipmunks (Tamias amoenus) as important vertebrate reservoirs for the enzootic transmission of POWV (McLean and Larke 1963). The red squirrels and chipmunks were frequently infested with I. marxi (McLean and Larke 1963). Ensuing surveillance efforts demonstrated that groundhogs (Marmota monax) had relatively high rates of neutralizing antibodies to POWV and that they were frequently infested with I. cookei (McLean et al. 1964, 1967). Mustelids, specifically skunks, are also thought to be involved in the enzootic maintenance of POWV (Main et al. 1979, Johnson 1987).

The initial isolates of DTV from $I$. scapularis ticks suggested that white-footed mice (Peromyscus leucopus) were likely to be involved in the natural transmission cycles of DTV (Telford et al. 1997) (Fig. 1). A study conducted in both Wisconsin and New England demonstrated that in areas with dense levels of DTV-infected I. scapularis, 3\%-4\% of collected mice were seropositive for DTV (Ebel et al. 2000). This implicated white-footed mice as reservoir hosts for DTV. Furthermore, when the seroprevalence for POWV was tested in white-tailed deer (often parasitized by adult $I$. scapularis), these animals proved to be useful sentinels for assessing the enzootic activity of POWV and DTV (Nofchissey et al. 2013). In North America, there appear to be three main enzootic cycles occurring: I. marxi and squirrels, I. cookei and groundhogs/mustelids, and I. scapularis and white-footed mice. Because I. scapularis tend to feed on a variety of hosts, including white-footed mice, it is likely that some enzootic cycles of POWV occur between I. scapularis and other mammals such as voles (Myodes sp). In one surveillance study, several species of voles were seropositive for POWV (Deardorff et al. 2013).

The potential of Ixodes scapularis to drive further emergence of Powassan encephalitis

The host specificity, questing behavior, and nidicolous (nest or burrow dwelling) tendencies of a tick species all determine the intensity of enzootic transmission and the likelihood of the tick transmitting POWV or DTV to humans.
The I. cookei tick, which frequently parasitizes groundhogs and mustelids, is believed to be nidicolous and does not exhibit questing behavior (Ko 1972). Generations of I. cookei are closely associated with a single host in its burrow, so humans are less likely to come into contact with POWVinfected I. cookei (Hinten et al. 2008, Ebel 2010). Except for parts of Maine where I. cookei appear to parasitize humans more readily than in other states (Smith et al. 1992, Rand et al. 2007), POWV is often maintained in a primarily enzootic cycle between groundhogs/mustelids and I. cookei. Conversely, I. scapularis are not as host specific as I. cookei and are non-nidicolous, relying heavily on leaf litter to prevent desiccation and provide protection. Unlike I. cookei, I. scapularis will aggressively bite hosts such as white-footed mice, white-tailed deer, and even humans. Thus, I. scapularis may pose a greater public health threat than other Ixodes species since they are more likely to parasitize humans and they can vector DTV and other human pathogens, including Borrelia burgdorferi, Anaplasma phagocytophilum, Babesia microti, and the recently identified Borrelia miyamotoi (Wroblewski et al. 2017). A recent survey of I. scapularis distribution throughout the United States demonstrated that two previously distinct foci for I. scapularis (in the Northeast and Midwest) seem to be merging in the Ohio River Valley, creating one continuous focus (Eisen et al. 2016). Together, the aggressive feeding nature, catholic host preference, and geographic expansion of I. scapularis suggest that it has more potential than other Ixodes species to drive further emergence of POWV encephalitis in North America.

Laboratory studies have demonstrated the vector competence of several tick species for POWV, including I. scapularis. As with TBEV, a tick may become infected with POWV at various feeding points in its life cycle by horizontal transmission, transstadial transmission, or vertical transmission. Although it is believed that $D$. andersoni do not play a major role in natural transmission cycles of POWV, laboratory studies have shown that it is a competent vector species (Chernesky 1969, Chernesky and McLean 1969). In 1996, Costero and Grayson demonstrated under experimental conditions that I. scapularis is a competent vector of POWV (Lineage I). Horizontal transmission of POWV from viremic hamsters and rabbits to uninfected I. scapularis occurred, as did transstadial transmission (Costero and Grayson 1996). Vertical transmission of POWV did not occur from infected D. andersoni female adults to eggs (Chernesky 1969), but it was observed with I. scapularis (Costero and Grayson 1996).

\section{Clinical Disease Overview}

\section{The course of human disease}

In humans, POWV is the cause of a rare but severe neuroinvasive disease. The incubation period can range from 1 to 5 weeks, but most patients are unable to pinpoint the date when they were exposed (Ebel 2010). The majority of symptomatic POWV cases typically involve an initial febrile illness (Fig. 2). During the prodromal phase, sore throat, drowsiness, headache, and disorientation are commonly present (Smith et al. 1974). In more severe cases that progress to neurological involvement, the most common clinical presentations of disease are encephalitis, meningoencephalitis, and aseptic meningitis. The encephalitic phase is characterized by vomiting, prolonged fever, respiratory distress, loss 


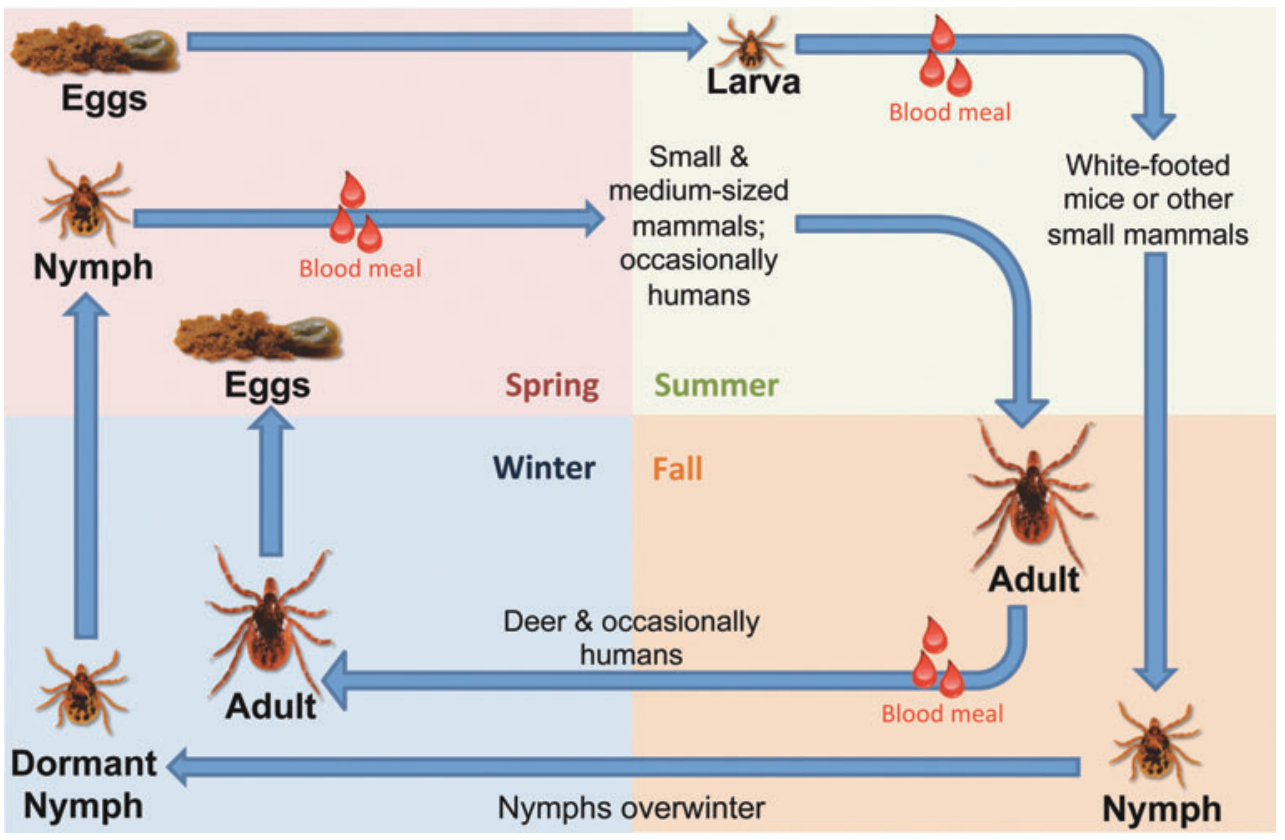

FIG. 1. Life cycle and feeding stages of Ixodes scapularis. I. scapularis is a three-host tick that requires approximately 2 years to complete its life cycle. I. scapularis larvae typically feed in the late summer months (Year 0), promptly molting and overwintering as unfed nymphs. In May to July of the following year (Year 1), the nymphs feed and molt to adults. Adult $I$. scapularis begin questing from approximately October of Year 1 to April of Year 2, with replete females laying eggs in the late spring (Year 2) that hatch by August, completing the 2-year life cycle.

Timeline of infection:

\begin{tabular}{|l|c|}
\hline $\begin{array}{c}1 \text { to } 5 \text { week } \\
\text { incubation period }\end{array} \quad$ Approximately 1 week $\quad$ Weeks to months \\
\hline Infection
\end{tabular}

\section{Symptoms:}

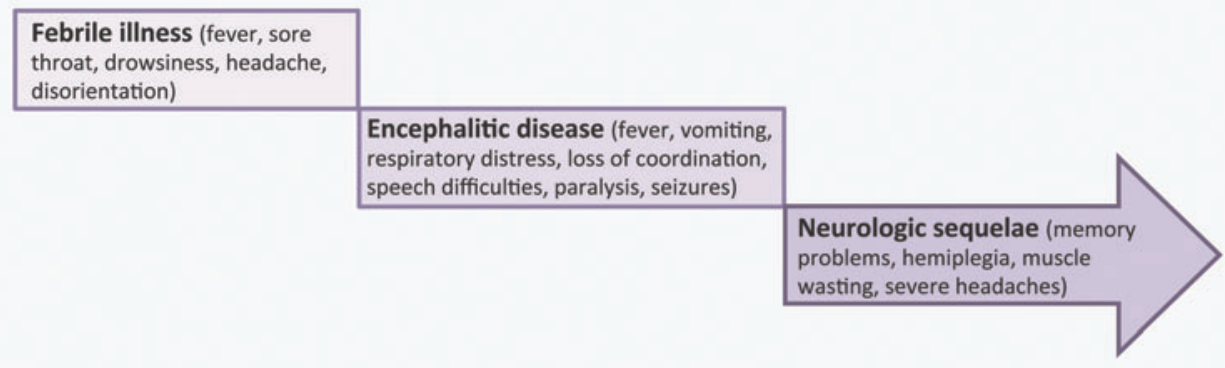

\section{Diagnostic testing:}

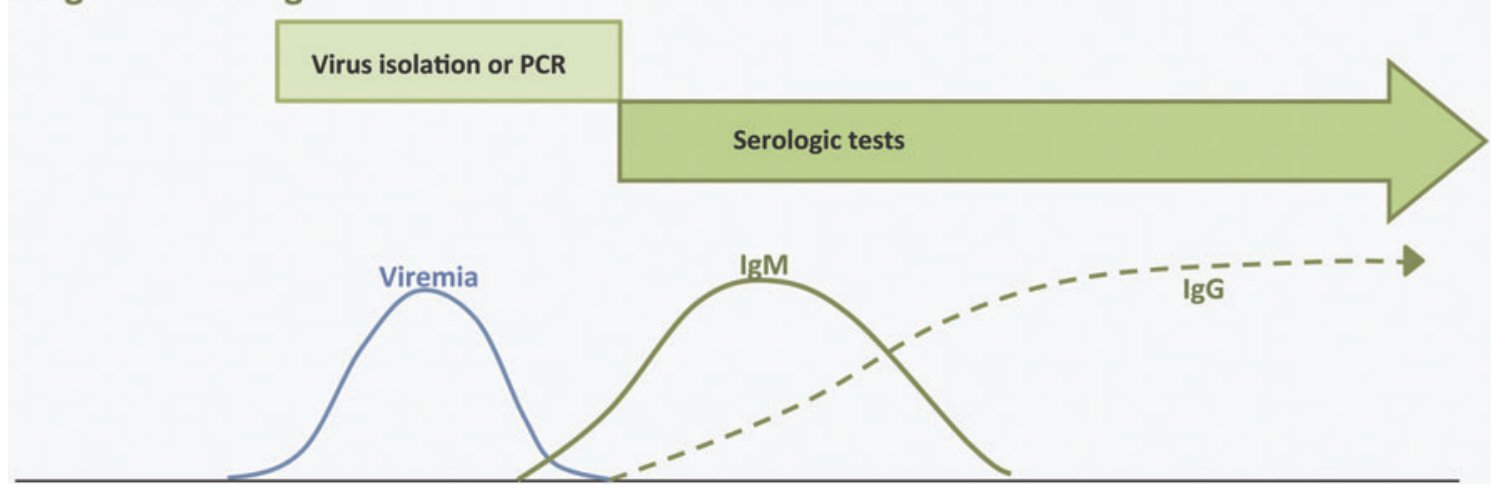

FIG. 2. Timeline of POWV infection, symptoms, and diagnostic testing. Shown is the chronology of POWV infection in relation to the clinical signs and symptoms of disease. During the early, viremic phase of disease, POWV can be diagnosed by virus isolation or by detection of specific nucleic acid/viral antigen. At later stages of disease, POWV-specific IgM and IgG antibodies can be detected. POWV, Powassan virus. 
of coordination, difficulty speaking, and seizures (Smith et al. 1974) (Fig. 2). Ocular symptoms, including ophthalmoplegia (Lessell and Collins 2003) and direction-changing nystagmus, have been reported in some cases of POWV encephalitis (Piantadosi et al. 2016). Throughout the encephalitic phase of disease, lethargy and some degree of paralysis are typical (Smith et al. 1974). Approximately 10\% of POWV encephalitis cases are fatal, and severe and long-lasting neurological sequelae are present in over $50 \%$ of survivors (Ebel 2010). In a review of several early POWV encephalitis cases, hemiplegia is documented as the most common manifestation of neurologic damage (Smith et al. 1974). Other neurological sequelae include muscle wasting (Deibel et al. 1974), recurrent acute headaches (Goldfield et al. 1973), and memory problems (Fitch and Artsob 1990).

Throughout the literature there are reports of both spastic and flaccid paralyses in human infections with POWV (Santos et al. 2016). For the index case of POWV, McLean and Donahue described a spastic hemiplegia in the 5-year-old patient who succumbed to this disease (McLean and Donahue 1959). In 1972, another young child infected with POWV displayed signs of spastic paralysis, but ultimately survived the infection with moderate sequelae (Rossier et al. 1974). There were several other documented cases in children where rigidity in the limbs and spastic paralysis were detected, but the patients survived the infection and displayed mild to moderate neurologic sequelae (Wilson et al. 1979, Hicar et al. 2011). In contrast, several adult patients diagnosed with POWV displayed flaccid paralysis and areflexia during the acute phase of infection (Gholam et al. 1999, Hinten et al. 2008, Birge and Sonnesyn 2012). Based on the current data, it is unclear whether there is an age dependency for POWVinduced spastic versus flaccid paralysis (Santos et al. 2016). The type of paralysis observed during POWV infection likely depends on the region(s) of the brain and spinal cord that are most severely impacted by POWV infection, inflammatory cell infiltration, and necrosis.

Since Ebel's 2010 POWV review, hemorrhagic encephalitis was described in two additional POWV cases. In one of these cases, a 43-year-old male from Minnesota presented with bilateral thalamic hemorrhages, which resolved five months after the patient's hospital discharge (Choi et al. 2012). Another case series (also conducted in the midwestern United States) described a 60-year-old male POWV encephalitis patient with bilateral frontal and temporal intraparenchymal hemorrhage that resolved after 2 months (Raval et al. 2012).

\section{Pathological findings}

Including the index case, postmortem histopathological findings have been described in detail for three fatal human POWV cases and summarized in a review of POWV literature published by Ebel in 2010 (McLean and Donahue 1959, Gholam et al. 1999, Tavakoli et al. 2009, Ebel 2010). In summary, POWV encephalitis cases are typically characterized by perivascular and focal parenchymal infiltration composed largely of lymphocytes and monocytes (McLean and Donahue 1959, Gholam et al. 1999, Tavakoli et al. 2009). Occasional brain necrosis is associated with areas of more intense inflammatory infiltrates (Gholam et al. 1999). The widespread destruction of neuronal cells has been detected in the large motor neurons of the brainstem, spinal anterior horns, cerebellum, basal ganglia, and thalamus (Tavakoli et al. 2009). In addition, immunohistochemical detection of POWV antigen is marked in neuronal cell bodies and their processes, underscoring the neurotropism of this virus (Tavakoli et al. 2009).

There are few descriptions of spinal cord pathology for human POWV infections. For the index case of POWV, the cervical and lumbar regions of the spinal cord were examined, and mild inflammatory infiltrates were detected in both regions (McLean and Donahue 1959). A more detailed description of spinal cord pathology was provided by Tavakoli et al. (2009), where sequencing confirmed that the patient was infected with DTV. In this case report, mononuclear infiltrates were accentuated in the spinal anterior horns, with focal microglial nodules present in the lateral corticospinal tract and posterior column.

As with TBEV cases, MRI abnormalities for POWV cases are nonspecific and not diagnostic (Lindquist and Vapalahti 2009); however, in recent years, there has been an increase in POWV case studies that include descriptions of MRI findings, and such data contribute to the overall picture of POWV pathogenesis (Lessell and Collins 2003, Hinten et al. 2008, Tavakoli et al. 2009, Hicar et al. 2011, Birge and Sonnesyn 2012, Raval et al. 2012, El Khoury et al. 2013a, Sung et al. 2013, Piantadosi et al. 2016). The majority of the reported MRI findings for patients diagnosed with POWV describe T2/fluid-attenuation inversion recovery (FLAIR) hyperintensities within the brainstem, extending to the cortex and deep gray structures (Piantadosi et al. 2016). FLAIR is a pulse sequence used in MRIs, so a T2/FLAIR hyperintensity highlights an area of tissue that is abnormal compared to the surrounding tissue. A computerized tomography (CT) scan suggested intraparenchymal hemorrhage in one POWV patient, which was resolved at a follow-up, and MRI scans were not performed for that case (Raval et al. 2012).

\section{Animal models of pathogenesis}

Animal models are valuable experimental tools that can help elucidate the pathophysiology of a human disease. Ideally, an animal model should reflect similar disease manifestations and pathogenesis to the human disease. An overview of the various animals used in early POWV experiments is provided in Ebel's POWV review. Briefly, experiments with hamsters, monkeys, mice, chickens, rabbits, horses, and goats have each provided some additional information about POWV pathogenesis (Ebel 2010). Early POWV experiments with nonhuman primates showed clear evidence of the neurotropism of POWV, with neurons and glial cells as the preferential targets of infection (Frolova et al. 1985). In recent years, there have been relatively few reports of POWV small animal model development, especially compared to other tick-borne flaviviruses such as TBEV.

Suckling mice were used to generate some of the early isolates of POWV and DTV (McLean and Donahue 1959, Telford et al. 1997). Six-week-old BALB/c mice have also been used to model the course of infection and the timeline of POWV transmission (Ebel and Kramer 2004, Holbrook et al. 2005, Hermance and Thangamani 2015). When $10^{4}$ plaqueforming units (PFU) of POWV (LB strain) were intraperitoneally inoculated into 6-week-old female BALB/c mice, the first clinical signs of disease occurred at 5-6 days postinfection (dpi), the average survival time was 8 days, and all 
mice succumbed to the infection (Holbrook et al. 2005). When POWV-infected I. scapularis ticks (titers ranging from $\sim 10^{2}$ to $10^{4}$ PFU of POWV) were fed on 6-week-old female $\mathrm{BALB} / \mathrm{c}$ mice, the first signs of disease occurred 5 days after tick attachment, with a $100 \%$ mortality rate (Ebel and Kramer 2004). Similar clinical observations were made when BALB/ $\mathrm{c}$ mice of the same age and gender were inoculated in the hind footpad with $10^{3}$ PFU of POWV (LB strain) plus I. scapularis salivary gland extract (SGE) (Hermance and Thangamani 2015). The BALB/c mice in all three studies showed general signs of febrile illness (ruffled fur, hunched posture, and lethargy), which were followed by a rapid onset of the neurologic illness characterized by ataxia, loss of balance, hind limb paralysis, and death (Ebel and Kramer 2004, Holbrook et al. 2005, Hermance and Thangamani 2015). Four-week-old male C57BL/6 mice were also used to investigate POWV infection and pathogenesis. When these mice were footpad inoculated with two different doses of POWV (LB strain), a similar disease progression was observed and the mortality rate was $100 \%$ (Santos et al. 2016).

Histological examination of the brain sections from BALB/c mice that succumbed to POWV infection showed a clear meningoencephalitis, focal infiltration of mononuclear cell infiltrates, and perivascular cuffing (Holbrook et al. 2005). Necrotic lesions were detected throughout the cerebra (with the exception of the cerebellar parenchyma), and there was extensive POWV localization in neurons, which remained morphologically normal (Holbrook et al. 2005). In the C57BL/6 mouse model of POWV infection, neuropathological analysis showed meningoencephalitis with perivascular mononuclear infiltrates and microglial activation, as well as a poliomyelitis-like syndrome with POWV-infected motor neurons detected at the ventral horn of the spinal cord (Santos et al. 2016). Death of motor neurons in the brainstem and spinal cord is the likely cause of flaccid paralysis observed in the mice, and these findings are consistent with the flaccid paralysis observed in some human cases of POWV (Santos et al. 2016).

During the initial experiments that isolated DTV, it was observed that adult white-footed mice (Peromyscus leucopus) survived infection with DTV and showed no clinical signs of disease (Telford et al. 1997). When 4-week-old $P$. leucopus mice were intracranially or intraperitoneally inoculated with $10^{3}$ PFU of POWV, no overt clinical signs of disease were observed and all mice survived to the experimental endpoint of $28 \mathrm{dpi}$ (Mlera et al. 2017). Intracranially inoculated $P$. leucopus seroconverted to POWV, and histopathological analysis demonstrated mild inflammatory responses, including microgliosis and lymphocytic perivascular cuffing, in the brain and spinal cord from 5 to $15 \mathrm{dpi}$ (Mlera et al. 2017). Unlike inbred laboratory strains of mice, $P$. leucopus mice restrict POWV replication and do not develop clinical signs of disease.

\section{Diagnosis}

A neuroinvasive case of POWV is confirmed when it meets both the clinical disease criteria and one or more of the laboratory criteria for diagnosis (El Khoury et al. 2013a). A clinically compatible case of POWV neuroinvasive disease is defined as having a fever of $\geq 38^{\circ} \mathrm{C}$, any signs of peripheral or central nervous system dysfunction documented by a physi- cian, and the absence of a more likely clinical explanation (CDC Arboviral Disease Case Definition 2015). In addition to meeting the clinical disease criteria, one or more of the following laboratory criteria must be met for POWV diagnosis: POWV isolation; or detection of specific nucleic acid/ viral antigen in blood, CSF (cerebrospinal fluid), tissue, or other body fluids; or $\geq 4$-fold change in POWV-specific quantitative antibody titers in paired serum; or POWVspecific IgM antibodies in CSF with a negative result for other IgM antibodies in CSF for arboviruses endemic to the region where exposure occurred; or POWV-specific IgM antibodies in serum with confirmatory POWV-specific neutralizing antibodies in the same or a later specimen (CDC Arboviral Disease Case Definition 2015).

Virus isolation and detection of POWV RNA from blood and CSF samples can be used as diagnostic tools during the initial viremic phase of disease before seroconversion (Fig. 2). However, hospitalization of POWV cases usually occurs during the encephalitic phase of disease when POWV is cleared from the blood and CSF, and POWV-specific IgM and $\mathrm{IgG}$ antibodies are formed; thus, once the viremia is cleared, laboratory diagnostics are based on serologic tests. Solid-phase assays that detect POWV IgG and IgM antibodies commonly involve the enzyme-linked immunosorbent assay, immunofluorescence assay, and microsphere immunoassay. Such assays provide a presumptive diagnosis for POWV infection and should be followed by confirmatory serologic testing, which involves detection of POWVspecific neutralizing antibodies using an assay such as the plaque reduction neutralization test (CDC Arboviral Disease Case Definition 2015). During diagnosis, the patient's vaccination and travel histories should be considered, as well as potentially cross-reactive flaviviruses known to be transmitted in the same region of the patient's exposure.

Although serologically indistinguishable, POWV and DTV can be differentiated by using PCR primers specific to the envelope coding region or the NS5 coding region of each virus (El Khoury et al. 2013b). It is likely that the majority of cases reported to be POWV were not diagnosed using PCR testing to differentiate between the two genetic lineages; thus, an unknown portion of previously reported POWV cases may have instead been due to DTV infection (El Khoury et al. 2013b).

\section{Treatment and Prevention}

Presently, no vaccine or specific therapy is available to prevent or treat POWV infections. Thus, minimizing exposure to ticks is the best preventative measure for POWV infection. Individuals living in POWV-endemic areas are advised to wear light-colored clothing with pants tucked into socks, use tick repellants that contain DEET or permethrin, and to frequently search for and carefully remove any attached ticks from the body (CDC Preventing Tick Bites 2016). For POWV cases requiring hospitalization, supportive care is provided, and it often involves respiratory support, intravenous fluids, and reducing cerebral edema. There are several reports of high-dose corticosteroids used to treat patients with severe, neuroinvasive POWV disease, and all of the treated patients survived (Hicar et al. 2011, El Khoury et al. 2013a, Sung et al. 2013). Yet, the potential role that corticosteroid treatment plays in POWV disease outcome still 
remains unclear. Piantadosi et al. were the first to report the use of intravenous immunoglobulin (IVIG) for treatment of POWV encephalitis. Both patients treated with IVIG survived the POWV infection, although one patient displayed significant neurological sequelae after discharge (Piantadosi et al. 2016). The role of antiviral therapy in treating POWV disease is also unknown. One patient diagnosed with acute DTV encephalitis was treated with pegylated interferon alpha and ribavirin for three weeks, yet still succumbed to disease (El Khoury et al. 2013b).

\section{Recent Developments in POWV Research}

\section{Modeling intrahost population dynamics}

There is relatively low genetic diversity of POWV within field-collected I. scapularis, especially when compared to the intrahost diversity for mosquito-borne flaviviruses, such as West Nile virus (Brackney et al. 2010). Recently, the intrahost population dynamics for POWV was modeled in the laboratory. For these experiments, BALB/c mice were intraperitoneally infected with POWV and fed on by I. scapularis larvae and nymphs. The viral populations rapidly diversified within the mice; however, during horizontal transmission to ticks, the POWV populations were subject to severe transmission bottlenecks (Brackney and Armstrong 2016, Grubaugh et al. 2016). During transstadial transmission of POWV from larvae to nymphs, the transmission bottlenecks were relaxed (Brackney and Armstrong 2016, Grubaugh et al. 2016). Furthermore, strong purifying selection in ticks constrains the impact of RNAi-mediated diversification of POWV (Grubaugh et al. 2016). These laboratory studies were the first to examine the intrahost population dynamics for a tick-borne flavivirus, and several findings were in contrast to the evolutionary forces known to shape mosquitoborne flavivirus populations.

\section{Early timeline of POWV transmission}

The duration of I. scapularis attachment required for successful transmission of DTV to a host was found to be as little as 15 min (Ebel and Kramer 2004). This finding was particularly striking because unlike other tick-borne pathogens (Borrelia burgdorferi, Babesia microti, and Anaplasma phagocytophilum), very little time between tick attachment and virus transmission is needed for POWV. The reactivation period required for some nonviral tick-borne pathogens provides a grace period of approximately $24 \mathrm{~h}$, where a minimal risk of transmission occurs if humans remove the attached tick within this timeline; however, there is no such grace period for POWV due to its very short timeline of transmission. These differences underscore why the timeline of POWV transmission must be carefully considered when analyzing the early immunomodulatory events that occur at the feeding site of the tick.

\section{Host target cells during early stages of POWV infection}

Skin is a host's primary line of defense from the outside environment, and it is the first host organ that POWV and tick saliva encounter as the virus is delivered from the feeding tick's salivary glands (Wikel 2013, Hermance et al. 2016). Several recent studies examined the early cutaneous immune response of BALB/c mice to POWV-infected I. scapularis feeding (using the POWV-LB strain). Cutaneous immune gene expression analysis demonstrated that after $3 \mathrm{~h}$ of POWV-infected I. scapularis nymph feeding, a complex proinflammatory environment was present in the skin, including significant upregulation of genes related to granulocyte recruitment, migration, and accumulation (Hermance and Thangamani 2014). Histopathological analyses performed at the POWV-infected and POWV-uninfected I. scapularis feeding sites identified POWV antigen in macrophages and fibroblasts, providing the first report of cell types that are likely early targets for POWV infection in the skin (Hermance et al. 2016). In addition, histopathology showed that neutrophil and mononuclear cell infiltrates were recruited earlier to the POWV-infected tick feeding site versus the POWV-uninfected tick feeding site (Hermance et al. 2016). These studies with POWV-infected I. scapularis are useful for establishing a basic understanding of the intricate interactions between initial tick-mediated immunomodulation and the host immune response.

\section{Ixodes scapularis salivary factors facilitate POWV transmission and neuroinvasion}

It is widely accepted that tick saliva contains an array of pharmacologically active compounds that are vital to overcoming the coagulation, hemostasis, wound healing, and innate and adaptive immune responses of the host (Kazimírová and Štibrániová 2013, Wikel 2013). Saliva-assisted transmission (SAT) is the process by which bioactive salivary factors in tick saliva modulate the host environment, promoting transmission and establishment of the tick-borne pathogen. This phenomenon has been reported for several viruses, including TBEV and Thogoto virus, as well as other tick-borne pathogens (Jones et al. 1989, Labuda et al. 1993, Nuttall and Labuda 2004). The influence of I. scapularis saliva on POWV transmission and dissemination was recently demonstrated in BALB/c mice. A footpad coinoculation of $10^{3}$ PFU POWV (LB strain) plus I. scapularis SGE enhanced POWV transmission, dissemination, and neuroinvasion, ultimately resulting in meningoencephalitis and death for all mice (Hermance and Thangamani 2015). Conversely, mice infected with $10^{3}$ PFU POWV in the absence of SGE displayed no clinical signs of infection, no POWV immunostaining in the brain, and none succumbed to disease. When inoculated with $10^{6}$ PFU POWV, both with and without SGE, neuroinvasion and death occurred for all mice, and there were no differences in survival times, weight loss, clinical signs of infection, or patterns of virus dissemination between these groups (Hermance and Thangamani 2015). Thus, the phenomenon of SAT was demonstrated at the $10^{3}$ PFU dose of POWV but not at the $10^{6}$ PFU dose, suggesting that the effect of SGE on the course of disease is virus dose dependent. These studies demonstrated that following a low dose of POWV, I. scapularis SGE enhances virus transmission and neuroinvasion, but clearly a great deal more work is needed to identify the responsible bioactive salivary factors and to determine how they function during tick feeding to influence the SAT of POWV.

\section{Conclusions and Future Research Priorities}

Several recent studies suggested that the disease incidence of human POWV cases is rising in the United States (Hinten 
et al. 2008, Piantadosi et al. 2016). There is a growing possibility that POWV, like other I. scapularis-vectored pathogens, is an emerging human disease in regions with enzootic cycles of POWV and where I. scapularis are abundant (Nofchissey et al. 2013). Yet, with the overall lack of public awareness and infrequency of POWV testing in patients presenting with symptoms of encephalitis, the actual incidence of POWV cases is likely underestimated. Recently, it was shown that some tick populations in Wisconsin are coinfected with B. burgdorferi and DTV, suggesting that DTV coinfections may be underdiagnosed and could contribute to the persistent symptoms associated with some Lyme disease cases (Knox et al. 2015). The effect of Borrelia coinfection on POWV transmission is not well studied and should be addressed in future research.

It will be beneficial to pursue long-term vector and host surveillance in areas where human POWV cases have been identified. Screening in both adult and immature ticks collected from the field will provide a better understanding of the role each tick life stage plays in enzootic POWV transmission cycles. Furthermore, field surveillance programs will likely provide additional POWV strains, which will be instrumental in characterizing the evolutionary forces that shape POWV populations (Ebel 2010, Brackney and Armstrong 2016).

Currently, there is a limited understanding of the host antiviral response to POWV infection; therefore, future laboratory studies should examine the POWV replication cycle within mammalian and tick cells. By pursuing this knowledge gap, future studies can begin to evaluate new targets for therapeutic intervention. One of the major challenges in this field, and a future priority for POWV research, is the identification of factors that enable POWV to persist in nature while promoting human infection and pathogenicity. Consequently, it is crucial to determine the molecular mechanisms of tick-host-POWV interactions.

To better understand the neurological sequelae observed in many POWV patients, additional cohort studies with extended follow-up times should be conducted. The majority of POWV strains currently available for molecular genomics were isolated from ticks; therefore, laboratories that are equipped to isolate POWV from clinical samples should be encouraged to do so. Additional isolates of POWV derived from human tissue will enable comparative pathogenicity studies, which will be particularly useful in determining whether there are differences between POWV and DTV pathogenesis.

Along with the apparent rise in POWV cases has come a steady increase in the basic and applied research for POWV disease. In the upcoming years it will be important to maintain this research momentum and to increase clinician vigilance and public awareness.

\section{Acknowledgments}

S.T. is supported by the following grants: NIH/NIAID R01AI127771 and NIH/NIAID R21AI113128.

\section{Author Disclosure Statement}

No competing financial interests exist.

\section{References}

Anderson JF, Armstrong PM. Prevalence and genetic characterization of Powassan virus strains infecting Ixodes scapularis in Connecticut. Am J Trop Med Hyg 2012; 87:754-759.

Artsob H. Powassan encephalitis. In: Monath, TP, ed. The Arboviruses: Epidemiology and Ecology. Boca Raton, FL: CRC Press, 1989:29-49.

Beasley DW, Suderman MT, Holbrook MR, Barrett AD. Nucleotide sequencing and serological evidence that the recently recognized deer tick virus is a genotype of Powassan virus. Virus Res 2001; 79:81-89.

Birge J, Sonnesyn S. Powassan virus encephalitis, Minnesota, USA. Emerg Infect Dis 2012; 18:1669-1671.

Brackney DE, Armstrong PM. Transmission and evolution of tick-borne viruses. Curr Opin Virol 2016; 21:67-74.

Brackney DE, Brown IK, Nofchissey RA, Fitzpatrick KA, et al. Homogeneity of Powassan virus populations in naturally infected Ixodes scapularis. Virology 2010; 402:366-371.

Brackney DE, Nofchissey RA, Fitzpatrick KA, Brown IK, et al. Stable prevalence of Powassan virus in Ixodes scapularis in a northern Wisconsin focus. Am J Trop Med Hyg 2008; 79:971973.

Calisher CH, Karabatsos N, Dalrymple JM, Shope RE, et al. Antigenic relationships between flaviviruses as determined by cross-neutralization tests with polyclonal antisera. J Gen Virol 1989; 70:37-43.

Centers for Disease Control and Prevention. Arboviral Disease Case Definition. 2015. Available at https://wwwn.cdc.gov/ nndss/conditions/powassan-virus-disease/case-definition/2015

Centers for Disease Control and Prevention. Preventing tick bites. 2016. Available at www.cdc.gov/ticks/avoid/on_people .html

Chernesky MA, McLean DM. Localization of Powassan virus in Dermacentor andersoni ticks by immunofluorescence. Can J Microbiol 1969; 15:1399-1408.

Chernesky MA. Powassan virus transmission by ixodid ticks infected after feeding on viremic rabbits injected intravenously. Can J Microbiol 1969; 15:521-526.

Choi EE, Taylor RA. A case of Powassan viral hemorrhagic encephalitis involving bilateral thalami. Clin Neurol Neurosurg 2012; 114:172-175.

Costero A, Grayson MA. Experimental transmission of Powassan virus (Flaviviridae) by Ixodes scapularis ticks (Acari: Ixodidae). Am J Trop Med Hyg 1996; 55:536-546.

Deardorff ER, Nofchissey RA, Cook JA, Hope AG, et al. Powassan virus in mammals, Alaska and New Mexico, U.S.A., and Russia, 2004-2007. Emerg Infect Dis 2013; 19:2012-2016.

Deibel R, Flanagan TD, Smith V. Central nervous system infections in New York State. Etiologic and epidemiologic observations, 1974. N Y State J Med 1974; 75:2337-2342.

Ebel GD, Campbell EN, Goethert HK, Spielman A, et al. Enzootic transmission of deer tick virus in New England and Wisconsin sites. Am J Trop Med Hyg 2000; 63:36-42.

Ebel GD, Foppa I, Spielman A, Telford SR. A focus of deer tick virus transmission in the northcentral United States. Emerg Infect Dis 1999; 5:570-574.

Ebel GD, Kramer LD. Short report: Duration of tick attachment required for transmission of Powassan virus by deer ticks. Am J Trop Med Hyg 2004; 71:268-271.

Ebel GD, Spielman A, Telford SR III. Phylogeny of North American Powassan virus. J Gen Virol 2001; 82:1657-1665.

Ebel GD. Update on Powassan virus: Emergence of a North American tick-borne flavivirus. Annu Rev Entomol 2010; 55: 95-110. 
Eisen RJ, Eisen L, Beard CB. County-Scale Distribution of Ixodes scapularis and Ixodes pacificus (Acari: Ixodidae) in the Continental United States. J Med Entomol 2016; 53:349-386.

El Khoury MY, Camargo JF, White JL, Backenson BP, et al. Potential role of deer tick virus in Powassan encephalitis cases in Lyme disease-endemic areas of New York, U.S.A. Emerg Infect Dis 2013a; 19:1926-1933.

El Khoury MY, Hull RC, Bryant PW, Escuyer KL, et al. Diagnosis of acute deer tick virus encephalitis. Clin Infect Dis 2013b; 56:e40-e47.

Fitch W, Artsob H. Powassan encephalitis in New Brunswick. Powassan encephalitis in New Brunswick. Canadian Family Physician 1990; 33:1289-1290.

Frolova MP, Isachkova LM, Shestopalova NM, Pogodina VV. Experimental encephalitis in monkeys caused by the Powassan virus. Neurosci Behav Physiol 1985; 15:62-69.

Gholam BI, Puksa S, Provias JP. Powassan encephalitis: A case report with neuropathology and literature review. CMAJ 1999; 161:1419-1422.

Goldfield M, Austin SM, Black HC, Taylor BF, et al. A nonfatal human case of Powassan virus encephalitis. Am J Trop Med Hyg 1973; 22:78-81.

Grubaugh ND, Rückert C, Armstrong PM, Bransfield A, et al. Transmission bottlenecks and RNAi collectively influence tick-borne flavivirus evolution. Virus Evol 2016; 2:vew033.

Heinze DM, Gould EA, Forrester NL. Revisiting the clinal concept of evolution and dispersal for the tick-borne flaviviruses by using phylogenetic and biogeographic analyses. J Virol 2012; 86:8663-8671.

Hermance ME, Santos RI, Kelly BC, Valbuena G, Thangamani $\mathrm{S}$. Immune Cell Targets of Infection at the Tick-Skin Interface during Powassan Virus Transmission. PLoS One 2016; 11:e0155889.

Hermance ME, Thangamani S. Proinflammatory cytokines and chemokines at the skin interface during Powassan virus transmission. J Invest Dermatol 2014; 134:2280-2283.

Hermance ME, Thangamani S. Tick saliva enhances Powassan virus transmission to the host, influencing its dissemination and the course of disease. J Virol 2015; 89:7852-7860.

Hicar MD, Edwards K, Bloch K. Powassan virus infection presenting as acute disseminated encephalomyelitis in Tennessee. Pediatr Infect Dis J 2011; 30:86-88.

Hinten S, Beckett GA, Gensheimer KF, Pritchard E, et al. Increased recognition of Powassan encephalitis in the United States, 19992005. Vector Borne Zoonotic Dis 2008; 8:733-740.

Holbrook MR, Aronson JF, Campbell GA, Jones S, et al. An animal model for the tickborne flavivirus-Omsk hemorrhagic fever virus. J Infect Dis 2005; 191:100-108.

Johnson HN. Isolation of Powassan virus from a spotted skunk in California. J Wildl Dis 1987; 23:152-153.

Jones LD, Hodgson E, Nuttall PA. Enhancement of virus transmission by tick salivary glands. J Gen Virol 1989; 70:1895-1898.

Kazimírová M, Štibrániová I. Tick salivary compounds: Their role in modulation of host defenses and pathogen transmission. Front Cell Infect Microbiol 2013; 3:43.

Knox K, Thomm A, Harrington Y, Baewer D, et al. Arbovirus Co-Infections in Wisconsin Tick Populations. Open Forum Infect Dis 2015; 2(Suppl 1):1157.

Ko RC. Biology of Ixodes cookei Packard (Ixodidae) of groundhogs (Erxleben). Can J Zool 1972; 50:433-436.

Kuno G, Artsob H, Karabatsos N, Tsuchiya KR, et al. Genomic sequencing of deer tick virus and phylogeny of Powassanrelated viruses of North America. Am J Trop Med Hyg 2001; 65:671-676.
Kuno G, Chang GJ, Tsuchiya KR, Karabatsos N, et al. Phylogeny of the genus Flavivirus. J Virol 1998; 72:73-83.

Labuda M, Jones LD, Williams T, Nuttall PA. Enhancement of tick-borne encephalitis virus transmission by tick salivary gland extracts. Med Vet Entomol 1993; 7:193-196.

Leonova GN, Kondratov IG, Ternovoi VA, Romanova EV, et al. Characterization of Powassan viruses from Far Eastern Russia. Arch Virol 2009; 154:811-820.

Lessell S, Collins TE. Ophthalmoplegia in Powassan encephalitis. Neurology 2003; 60:1726-1727.

Lindquist L, Vapalahti O. Tick-borne encephalitis. Lancet 2009; 371:1861-1871.

Main AJ, Carey AB, Downs WG. Powassan virus in Ixodes cookei and Mustelidae in New England. J Wildl Dis 1979; 15: 585-591.

Mandl CW, Holzmann H, Kunz C, Heinz FX. Complete genomic sequence of Powassan virus: Evaluation of genetic elements in tick-borne versus mosquito-borne flaviviruses. Virology 1993; 194:173-184.

McLean DM, Best JM, Mahalingham S, Chernesky MA, et al. Powassan virus: Summer infection cycle, 1964. Can Med Assoc J 1964; 91:1360-1362.

McLean DM, Cobb C, Gooderham SE, Smart CA, et al. Powassan virus: Persistence of virus activity during 1966. Can Med Assoc J 1967; 96:660-664.

McLean DM, Crawford MA, Ladyman SR, Peers RR, et al. California encephalitis and Powassan virus activity in British Columbia, 1969. Am J Epidemiol 1970; 92:266-372.

McLean DM, Donahue W. Powassan virus: Isolation of virus from a fatal case of encephalitis. Can Med Assoc J 1959; 80: 708-711.

McLean DM, Larke RP. Powassan and Silverwater viruses: Ecology of two Ontario arboviruses. Can Med Assoc J 1963; 88:182-185.

Mlera L, Meade-White K, Saturday G, Scott D, et al. Modeling Powassan virus infection in Peromyscus leucopus, a natural host. PLoS Negl Trop Dis 2017; 11:e0005346.

Nofchissey RA, Deardorff ER, Blevins TM, Anishchenko M, et al. Seroprevalence of Powassan virus in New England deer, 1979-2010. Am J Trop Med Hyg 2013; 88:1159-1162.

Nuttall PA, Labuda M. Tick-host interactions: Saliva-activated transmission. Parasitology 2004; 129(Suppl):S177-S189.

Pesko KN, Torres-Perez F, Hjelle BL, Ebel GD. Molecular epidemiology of Powassan virus in North America. J Gen Virol 2010; 91:2698-2705.

Piantadosi A, Rubin DB, McQuillen DP, Hsu L, et al. Emerging Cases of Powassan Virus Encephalitis in New England: Clinical Presentation, Imaging, and Review of the Literature. Clin Infect Dis 2016; 62:707-713.

Rand PW, Lacombe EH, Dearborn R, Cahill B, et al. Passive surveillance in Maine, an area emergent for tick-borne diseases. J Med Entomol 2007; 44:1118-1129.

Raval M, Singhal M, Guerrero D, Alonto A. Powassan virus infection: Case series and literature review from a single institution. BMC Res Notes 2012; 5:594.

Rossier E, Harrison RJ, Lemieux B. A case of Powassan virus encephalitis. Can Med Assoc J 1974; 110:1173-1174.

Santos RI, Hermance ME, Gelman BB, Thangamani S. Spinal Cord Ventral Horns and Lymphoid Organ Involvement in Powassan Virus Infection in a Mouse Model. Viruses 2016; 8: pii:E220.

Schotthoefer A, Frost H, Thomm A, Dupuis A, et al. Evidence of High Rate of Powassan Virus Co-infection in Lyme Disease Patients. Open Forum Infect Dis 2016; 1:89. 
Smith R, Woodall JP, Whitney E, Deibel R, et al. Powassan virus infection. A report of three human cases of encephalitis. Am J Dis Childb 1974; 127:691-693.

Smith RP, Lacombe EH, Rand PW, Dearborn R. Diversity of tick species biting humans in an emerging area for Lyme disease. Am J Public Health 1992; 82:66-69.

Sung S, Wurcel AG, Whittier S, Kulas K, et al. Powassan meningoencephalitis, New York, New York, USA. Emerg Infect Dis 2013; 19:1504-1506.

Suss J. Tick-borne encephalitis 2010: Epidemiology, risk areas, and virus strains in Europe and Asia-an overview. Ticks Tick Borne Dis 2011; 2:2-15.

Tavakoli N, Wang H, Dupuis M, Hull R, et al. Fatal case of Deer tick virus encephalitis. N Engl J Med 2009; 60:2099_ 2107.

Telford SR, Armstrong P, Katavolos P, Foppa I, et al. A new tick-borne encephalitis-like virus infecting New England deer ticks, Ixodes dammini. Emerg Infect Dis 1997; 3:165-170.

Thomas LA, Kennedy RC, Ecklund CM. Isolation of a virus closely related to Powassan virus from Dermacentor andersonii collected along north Cache la Poudre River, Colo. Proc Soc Exp Biol Med 1960; 104:355-359.

U.S. Geological Survey Disease Maps: Powassan virus. Available at http://diseasemaps.usgs.gov/mapviewer

Wikel S. Ticks and tick-borne pathogens at the cutaneous interface: Host defenses, tick countermeasures, and a suitable environment for pathogen establishment. Front Microbiol 2013; 4:337.

Wilson MS, Wherrett BA, Mahdy MS. Powassan virus meningoencephalitis: A case report. Can Med Assoc J 1979; 121:320-323.

Wroblewski D, Gebhardt L, Prusinski MA, Meehan LJ, et al. Detection of Borrelia miyamotoi and other tick-borne pathogens in human clinical specimens and Ixodes scapularis ticks in New York State, 2012-2015. Ticks Tick Borne Dis 2017; 8:407-411.

Zanotto PM, Gould EA, Gao GF, Harvey PH, et al. Population dynamics of flaviviruses revealed by molecular phylogenies. Proc Natl Acad Sci U S A 1996; 93:548-553.

Zarnke RL, Yuill TM. Powassan virus infection in snowshoe hares (Lepus americanus). J Wildl Dis 1981; 17:303-310.

Address correspondence to: Saravanan Thangamani Department of Pathology University of Texas Medical Branch (UTMB) 301 University Boulevard Galveston, TX 77555-0609

E-mail: sathanga@utmb.edu 